\title{
Interleukin-6 Blocking vs. JAK-STAT Inhibition for Prevention of Lung Injury in Patients with COVID-19
}

\author{
Joseph Meletiadis (D) - Sotirios Tsiodras - Panagiotis Tsirigotis
}

Received: July 14, 2020 / Published online: August 12, 2020

(C) The Author(s) 2020

\begin{abstract}
The severe respiratory insufficiency observed during COVID-19 infection may not be directly related to a cytopathogenic effect induced by the virus itself, but to an exaggerated and inappropriate immune response. In an effort to reduce the severity of organ dysfunction, including respiratory insufficiency, monoclonal antibodies (Mabs) that block the interleukin-6 receptor, such as tocilizumab, sarilumab, and siltuximab, are under investigation for the treatment of COVID-19. However, blocking of just one of the many cytokines involved in the inflammatory reaction may not slow down the magnitude of the process. Since timing is important, the immune deficiency induced by
\end{abstract}

Digital Features To view digital features for this article go to https://doi.org/10.6084/m9.figshare.12728231.

J. Meletiadis $(\bowtie)$

Clinical Microbiology Laboratory, Attikon

University Hospital, Medical School, National and

Kapodistrian University of Athens, Athens, Greece

e-mail: jmeletiadis@med.uoa.gr

S. Tsiodras

Forth Department of Internal Medicine, Attikon University Hospital, Medical School, National and Kapodistrian University of Athens, Athens, Greece

P. Tsirigotis

Second Department of Internal Medicine, Attikon

University Hospital, Medical School, National and

Kapodistrian University of Athens, Athens, Greece
IL6 blockade at the late immunodeficiency phase of sepsis that follows the initial inflammatory response may be detrimental. Finally, monitoring the degree and duration of IL6 blockade may be challenging because of the long half-life of Mabs (2-3 weeks). Pro- and antiinflammatory cytokines act through a common JAK-STAT signaling pathway, which can be inhibited by JAK-STAT inhibitors. Ruxolitinib, a tyrosine kinase inhibitor selective for JAK1, 2, blocks many pro- and anti-inflammatory cytokines including IL6. Ruxolitinib has favorable pharmacodynamics and an acceptable safety profile. The short half-life (4-6 h) of the drug offers the opportunity for ideal monitoring of the degree and duration of cytokine blocking, simply by the adjusting dose and duration of therapy. From a theoretical point of view, the balanced control of cytokine blockade throughout the course of the septic process should be the cornerstone of modern management. According to this hypothesis, maximization of blocking should be attempted at the phase of hyper-inflammation for preventing severe organ damage, while pro-inflammatory blockade should be minimized at the late phase of immunoparalysis for prevention of secondary infections. Based on the above considerations, we consider that the efficacy and safety of this drug deserves testing in the context of a controlled randomized trial. 
Keywords: COVID-19; Cytokine storm; IL6 blocking; JAK-STAT inhibitors; Ruxolitinib; Sarilumab; Severe respiratory syndrome; Siltuximab; Tocilizumab

\section{Key Summary Points}

COVID-19 infection cause an exaggerated and inappropriate immune response.

Monoclonal antibodies blocking interleukin-6 receptor have been used to reduce this response.

Blocking one cytokine may not slow down the magnitude of the process, and monitoring the degree and duration of IL6 blockade may be challenging because of long half-life of those antibodies (2-3 weeks).

JAK-STAT inhibitors block many pro- and anti-inflammatory cytokines including IL6 and have favorable pharmacodynamics.

Because of its short half-life (4-6 h), the degree and duration of cytokine blocking can be easily monitored by adjusting the dose and duration of therapy.

\section{INTRODUCTION}

An unknown percentage of patients infected with COVID-19 progress to lower respiratory tract involvement usually complicated by severe acute respiratory distress syndrome (ARDS). It has been postulated that the severe respiratory insufficiency observed during the course of infection is not directly related to a cytopathogenic effect induced by the virus itself, but it is rather the result of an exaggerated and inappropriate immune response [1]. Therefore, in an effort to reduce the severity of organ damage, immune-modulating or immunosuppressive agents have been used by many physicians in critically ill patients, such as corticosteroids or monoclonal antibodies (Mabs). Among them, the Mabs tocilizumab, sarilumab, and siltuximab with specificity against interleukin-6 (IL6) are the most widely considered [2]. Anti-IL6 antibody administration is supported by data showing that a cytokine storm mimicking sepsis occurs during the course of this viral infection [1]. Further supporting the role of IL6 in the pathogenesis of COVID-19, a recent trial including 89 hospitalized patients showed that increased serum IL6 levels was the most accurate predictor for the subsequent need for mechanical ventilation [3].

Although data from controlled randomized trials are lacking, anecdotal experience suggests that anti-IL6 antibody use has at least modest activity in patients with severe respiratory dysfunction and concurrent COVID-19 infection. To our knowledge, among anti-IL6 antibodies, tocilizumab has been the most widely used. Tocilizumab is a Mab with specificity against the IL6-receptor and has been granted approval for the treatment of rheumatoid arthritis and Still's disease. Existing data regarding the safety and efficacy of tocilizumab are limited to single case or case series reports. In the largest published report, tocilizumab was administered in 15 patients with respiratory failure due to COVID-19 and showed promising efficacy [4]. In a previous commentary, $\mathrm{Fu}$ et al. presented the rationale behind the promising efficacy of tocilizumab as a treatment for the ARDS observed in patients with COVID-19 [5]. The timing of administration of this agent remains one of the most important parameters in assessing its beneficial effect.

Although much is known about the pathogenesis of the septic process induced by common bacteria, any extrapolation of these data to the infectious process induced by COVID-19 is arbitrary and should be interpreted with caution. Moreover, the pathogenesis of COVID-19 is extremely complex, and the concept of a single-cytokine blockade is over-simplistic. A recent trial examined the dynamic changes during the course of infection of lymphocyte subsets and cytokine profile in 40 patients with COVID-19. Progressive decrease in the counts of CD8 cells and increasing serum levels of IL6, IL10, 1L2, and IFN-g were strongly associated 
with disease severity and were excellent predictors of outcome [6]. This article is based on previously conducted studies and does not contain any studies with human participants or animals performed by any of the authors.

\section{THEORETICAL DISADVANTAGES OF IL6 BLOCKING}

Considering the existing experience of treatment with anti-IL6 monoclonal antibodies and the lack of solid data regarding COVID-19 pathogenesis, we assume that at least from a theoretical point of view anti-IL6 antibody administration may be associated with several drawbacks that are briefly discussed below. (1) The monoclonal antibodies that block IL6 like siltuximab rather than the IL6 receptor like tocilizumab and sarilumab inhibit the cis/transsignaling but not the trans-presentation via Th17 lymphocytes [7]. (2) The immune response and the resulting inflammatory reaction against a pathogen are usually driven by a multitude of cytokines, and therefore blocking of just one of the many is not anticipated to slow down the magnitude of the process, simply because the absence of function of one cytokine is counterbalanced by other pro-inflammatory mediators acting in parallel [8]. As a proof of the principle of the complexity of the septic process and the multiplicity of the cytokines involved, a recent study showed significant elevation in the levels of 38 out of 48 measured cytokines in the plasma of patients with COVID-19. More importantly, 17 cytokines showed significant correlation with the viral load, while 15 cytokines besides IL6 were strongly associated with the severity of lung injury [9]. In a similar trial including 50 patients with COVID-19 infection, serum levels of 14 cytokines were found to be significantly elevated in patients compared with healthy volunteers. Importantly, different disease severity was characterized by the specific cytokine expression profile. Furthermore, IFN- $\gamma$-induced protein 10 (IP10) and monocyte chemotactic protein-3 (MCP3) were the best predictors for progression to respiratory failure [10]. (3) Moreover, even if we assume that blocking IL6 will be sufficient to prevent severe lung damage, we should not underestimate the immune deficiency induced by IL6 blockade, especially if we consider the late immunodeficiency phase of sepsis that follows the initial inflammatory response (Fig. 1). (4) Another important disadvantage of anti-IL6 antibodies is the difficulty in monitoring the degree and duration of IL6 blockade. We should keep in mind that complete, and of prolonged duration, blockade of the inflammatory phase might have detrimental effects because of the inability to eliminate the pathogen and the subsequent termination of the immune process (Fig. 1). Mabs have a half-life of 2-3 weeks, and therefore reversal of cytokine blocking is not possible for a few weeks after administration [11]. Prolonged and/or intensive pro-inflammatory cytokine blockade may exacerbate the immune suppression that usually occurs at the late phase of sepsis [12]. As a result, patients hospitalized in ICU frequently develop secondary infections such as ventilator-associated pneumonia or bloodstream infections caused by weakly virulent pathogens such as Acinetobacter, Stenotrophomonas, Candida, etc. [13]. Secondary infections lead to repeated cycles of hyper- and hypo-inflammatory phases further complicating the septic process and not infrequently resulting in the death of the patient despite the elimination of the pathogen that triggered the initial septic event (Fig. 1). Administration of an anti-IL6 antibody for the treatment of hyperinflammation observed during COVID-19 infection, although initially reducing the elimination of the cytokine storm and possibly the need and duration of mechanical ventilation, may subsequently increase the risk of secondary infections and mortality (Fig. 1). Data regarding the incidence of secondary infections and death without COVID-19 in patients with COVID-19 treated with immunosuppressive agents such as anti-IL6 antibodies are absent. Administration of such agents in patients with infections is justified only in the context of well-designed prospective randomized trials. 

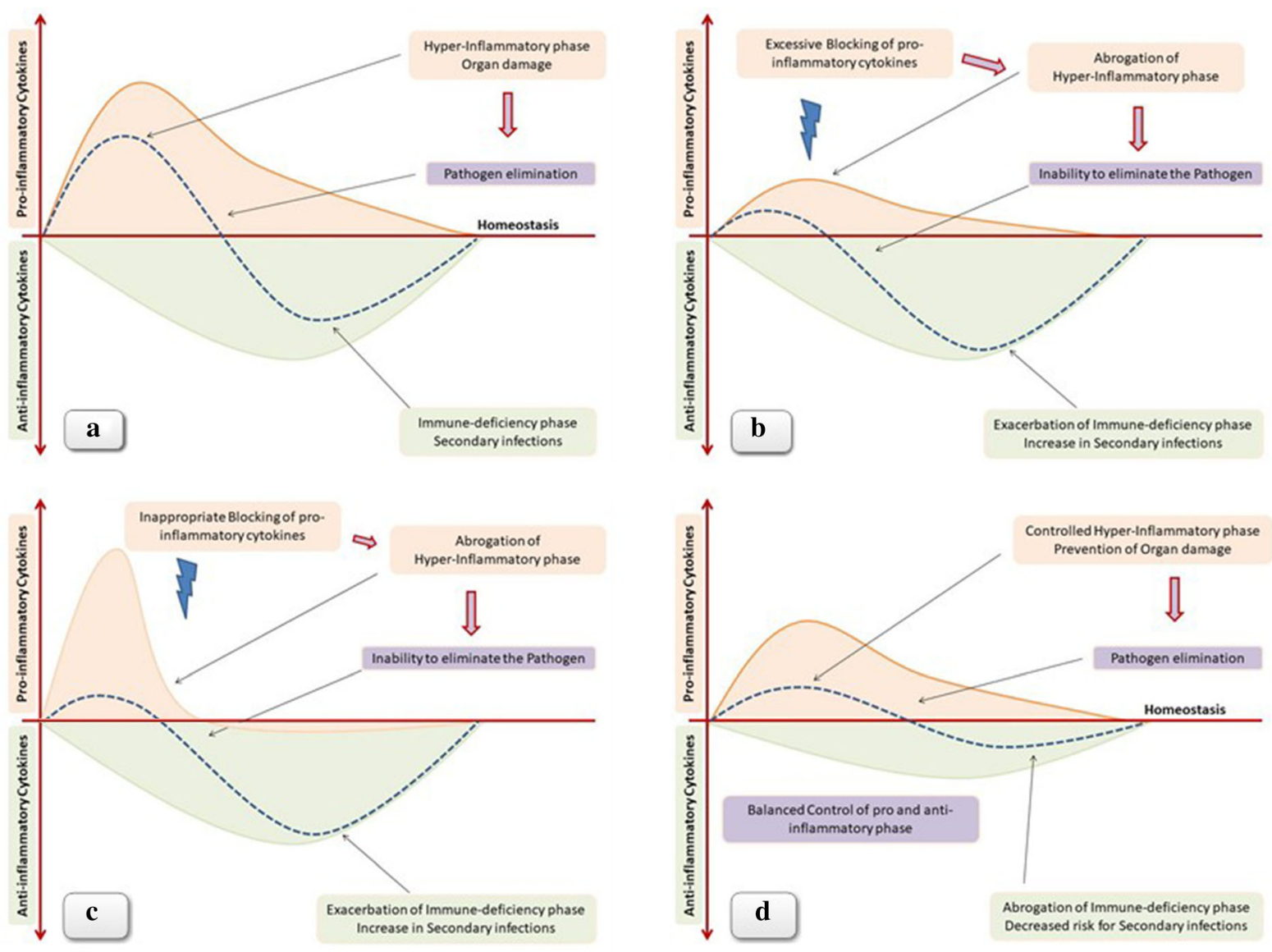

Fig. 1 a) Although oversimplified, the septic process is characterized by an initial hyper-inflammatory phase followed by a late phase of immnue-deficiency. Blocking of pro-inflammatory cytokines has been proposed as a therapeutic modality with the aim to prevent the cytokine strom and the resulting organ damage. However, either excessive b) or inappropriate and at the wrong time c) proinflammatory blockade may result in exacerbation of immune-deficiency, inability to eliminate the pathogen, and increased risk for secondary infections. d) Any

\section{ANTI-INFLAMMATORY EFFECT OF JAK-STAT PATHWAY INHIBITION}

Ruxolitinib is a Janus tyrosine kinase (JAK) inhibitor approved for the treatment of myelofibrosis and hydroxyurea refractory polycythemia-vera. Although ruxolitinib is effective in reducing spleen size, the beneficial therapeutic effect in myelofibrosis is mostly explained by its anti-inflammatory properties

immune-modulating therapeutic approach in sepsis should be applied in combination with an effective anti-pathogen agent. Future studies exploring immune-modulation in sepsis should take into account dynamic parameters such as the intensity, the duration, as well as the proper timing of immune intervention. Theoretically, an ideal approach should focus on a balanced control of both hyperinflammation and immune-suppression with the aim to prevent organ dysfunction but without compromising the ability of the immune system to eliminate the pathogen

[14]. Pro- and anti-inflammatory cytokines act through a common signaling pathway consisting of different JAK kinases interacting with signal transducer and activator of transcription (STAT) proteins. Ruxolitinib, by inhibiting JAK proteins, at least theoretically, induces a global immune modulation affecting not only the initial inflammatory but also the late immune deficiency phase of sepsis.

In previous experiments performed by our group, when using different doses of ruxolitinib 
in an animal model of sepsis due to candida, we observed that survival improvement occurred with intermediate dosing, while excessive or very low dosing had a detrimental or no effect on survival. Mice treated with high-dose ruxolitinib had very high fungal loads but very low inflammation scores compared with controls, meaning that intensive abrogation of the inflammatory phase results in uncontrolled pathogen proliferation. On the other hand, mice treated with the optimal dose of ruxolitinib had the same fungal load but a lower inflammation score compared with controls, meaning that increased survival is due to prevention of excessive tissue injury and not to an antifungal effect of the study drug [15].

In accordance to our findings, ruxolitinib showed significant efficacy in an animal model of hemophagocytic lymphohistiocytosis (HLH) induced by infection due to lymphocyte choriomeningitis virus (LCMV) [16]. Preliminary analysis of data of a clinical trial testing ruxolitinib as treatment for adult hemophagocytic syndrome has also shown promising results [17]. Of note, hemophagocytic syndrome is a hyper-inflammatory reaction characterized by acute respiratory distress, cytokine storm, cytopenia, and high ferritin and IL6 levels as observed in COVID-19 patients with severe respiratory failure.

\section{POTENTIAL ADVANTAGES AND DRAWBACKS OF JAK INHIBITION OVER IL6 BLOCKING}

Based on the above, it is reasonable to assume that ruxolitinib may be effective in patients with COVID-19 and lung injury, and the efficacy and safety of the drug deserves testing in the context of a controlled randomized trial. The theoretical benefits of ruxolitinib over an anti-IL6 antibody are discussed below. (1) Ruxolitinib blocks not only IL6 but also many proand anti-inflammatory cytokines inducing a global immune modulation. (2) The degree and duration of cytokine blocking can be easily monitored by using escalating dosing in different cohorts of patients and by increasing the days of administration. Avoiding of complete blocking of the initial hyper-inflammatory phase may also prevent a protracted and severe late immune deficiency phase [18]. (3) The short half-life of the drug (4-6 h) gives a significant advantage over any anti-IL6 antibody, since any possible harm due to immune suppression should be more easily reverted after drug discontinuation.

However, some theoretical drawbacks exist as well that may have important clinical implications. The JAK-STAT signaling process upregulates interferon-controlled genes, a pathway that is frequently inhibited by viral products resulting in increased virulence. Thus, such inhibition could facilitate viral replication as well as viral re-activation of either SARS-CoV-2 (if this is confirmed) or other latent viruses such as herpes simplex or herpes zoster viruses. Thus, anti-herpes prophylaxis should be considered in immunosuppressed individuals receiving such agents [19].

\section{DISCUSSION AND CONCLUSIONS}

The impact of the COVID-19 pandemic on the social health system has been devastating in many countries, and there is an unmet clinical need for effective treatments or therapeutic interventions. As a result, the safety and efficacy of various drugs have been tested in clinical trials, while many more drugs have been administered in a compassionate use in small cohorts of patients. However, interpretation of data from many clinical trials is inconclusive mainly because of the absence of a comparator arm. Tocilizumab has shown promising effects, and prospective randomized trials testing its efficacy and safety in patients with pneumonia due to COVID-19 are ongoing. In this commentary, we present our hypothesis regarding some of the theoretical advantages and disadvantages of JAK inhibition over IL6 blocking. Transgenic animals overexpressing ACE2 receptors could facilitate further examination of the role of such immunomodulation in COVID therapeutics [20]. Understanding the urgent need for therapeutic schemes for the severe respiratory failure manifestations of the new COVID-19 infectious disease, we believe that 
the administration of immunosuppressive agents should be implemented only in the context of controlled randomized trials that should consider the appropriate timing and dosage of such administration and other prophylaxis issues.

\section{ACKNOWLEDGEMENTS}

Funding. No funding or sponsorship was received for this study or publication of this article.

Authorship. All named authors meet the International Committee of Medical Journal Editors (ICMJE) criteria for authorship for this article, take responsibility for the integrity of the work as a whole, and have given their approval for this version to be published.

Authorship Contributions. Joseph Meletiadis, Sotirios Tsiodras and Panagiotis Tsirigotis have contributed to writing and reviewing the article. All authors read and approved the final manuscript.

Disclosures. Sotirios Tsiodras and Panagiotis Tsirigotis declare that they have no competing interests. Joseph Meletiadis is a member of the journal's Editorial Board.

Compliance with Ethics Guidelines. This article is based on previously conducted studies and does not contain any studies with human participants or animals performed by any of the authors.

Open Access. This article is licensed under a Creative Commons Attribution-NonCommercial 4.0 International License, which permits any non-commercial use, sharing, adaptation, distribution and reproduction in any medium or format, as long as you give appropriate credit to the original author(s) and the source, provide a link to the Creative Commons licence, and indicate if changes were made. The images or other third party material in this article are included in the article's Creative Commons licence, unless indicated otherwise in a credit line to the material. If material is not included in the article's Creative Commons licence and your intended use is not permitted by statutory regulation or exceeds the permitted use, you will need to obtain permission directly from the copyright holder. To view a copy of this licence, visit http://creativecommons.org/licenses/by$\mathrm{nc} / 4.0 /$.

\section{REFERENCES}

1. Huang C, Wang Y, Li X, et al. Clinical features of patients infected with 2019 novel coronavirus in Wuhan. China Lancet. 2020;395(10223):497-506.

2. Zhang C, Wu Z, Li JW, Zhao H, Wang GQ. The cytokine release syndrome (CRS) of severe COVID19 and interleukin-6 receptor (IL-6R) antagonist tocilizumab may be the key to reduce the mortality. Int J Antimicrob Agents. 2020 Mar;29:105954. https://doi.org/10.1016/j.ijantimicag.2020.105954.

3. Herold T, Jurinovic V, Arnreich C, et al. Elevated levels of IL-6 and CRP predict the need for mechanical ventilation in COVID-19. J Allergy Clin Immunol. 2020;146(1):128-136.e4.

4. Luo P, Liu Y, Qiu L, Liu X, Liu D, Li J. Tocilizumab treatment in COVID-19: a single center experience. J Med Virol. 2020;92:814-8.

5. Fu B, Xu X, Wei H. Why tocilizumab could be an effective treatment for severe COVID-19? J Transl Med. 2020;18(1):164.

6. Liu J, Li S, Liu J, et al. Longitudinal characteristics of lymphocyte responses and cytokine profiles in the peripheral blood of SARS-CoV-2 infected patients. EBioMedicine. 2020;55:102763.

7. Garbers C, Heink S, Korn T, Rose-John S. Interleukin-6: designing specific therapeutics for a complex cytokine. Nat Rev Drug Discov. 2018;17(6):395-412.

8. Kyriazopoulou E, Leventogiannis K, NorrbyTeglund A, et al. Macrophage activation-like syndrome: an immunological entity associated with rapid progression to death in sepsis. Hellenic Sepsis Study. Group BMC Med. 2017;15(1):172.

9. Liu Y, Zhang C, Huang F, et al. Elevated plasma levels of selective cytokines in COVID-19 patients reflect viral load and lung injury. Natl Sci Rev. 2020;7:1003-111. 
10. Yang Y, Shen C, Li J, et al. Plasma IP-10 and MCP-3 levels are highly associated with disease severity and predict the progression of COVID-19. J Allergy Clin Immunol. 2020;146(1):119-127.e4.

11. Sheppard M, Laskou F, Stapleton P, Hadavi S, Dasgupta B. Tocilizumab (Actemra). Hum Vaccin Immunother. 2017;13(9):1972-88.

12. Hotchkiss RS, Karl IE. The pathophysiology and treatment of sepsis. N Engl J Med. 2003;348:138-50.

13. Kollef KE, Schramm GE, Wills AR, et al. Predictors of 30- day mortality and hospital costs in patients with ventilator associated pneumonia attributed to potentially antibiotic resistant gram-negative bacteria. Chest. 2008;134:281-7.

14. Verstovsek S, Kantarjian H, Mesa R. Safety and efficacy of INCB018424, a JAK1 and JAK2 inhibitor, in myelofibrosis. N Engl J Med. 2010;363:1117-27.

15. Tsirigotis P, Papanikolaou N, Elefanti A, et al. Treatment of experimental candida sepsis with a Janus kinase inhibitor controls inflammation and prolongs survival. Antimicrob Agents Chemother. 2015;59(12):7367-73.
16. Albeituni S, Verbist KC, Tedrick PE, et al. Mechanisms of action of ruxolitinib in murine models of hemophagocytic lymphohistiocytosis. Blood. 2019;134(2):147-59.

17. Ahmed A, Merrill S, Alsawah F, et al. Ruxolitinib in adult patients with secondary haemophagocytic lymphohistiocytosis: an open-label, single-centre, pilot trial. Lancet Haematol. 2019;6(12): e630-e637637.

18. Tsirigotis P, Chondropoulos S, Gkirkas K, Meletiadis J, Dimopoulou I. Balanced control of both hyper and hypo-inflammatory phases as a new treatment paradigm in sepsis. J Thorac Dis. 2016;8(5): E312-E316316.

19. Fleming S. Viral inhibition of the IFN-Induced JAK/ STAT signalling pathway: development of live attenuated vaccines by mutation of viral-encoded IFN-antagonists. Vaccines. 2016;4:23.

20. Yang XH, Deng W, Tong Z, et al. Mice transgenic for human angiotensin-converting enzyme 2 provide a model for SARS coronavirus infection. Comp Med. 2007;57(5):450-9. 